\title{
Association between echo intensity and attenuation of skeletal muscle in young and older adults: a comparison between ultrasonography and computed tomography
}

This article was published in the following Dove Press journal:

Clinical Interventions in Aging

\section{Yuya Watanabe ${ }^{1-3}$ \\ Masahiro Ikenaga ${ }^{4}$ \\ Eiichi Yoshimura ${ }^{5}$ \\ Yosuke Yamada ${ }^{2,6}$ \\ Misaka Kimura ${ }^{2,3}$}

'Faculty of Health and Sports Science, Doshisha University, Kyotanabe, Japan; ${ }^{2}$ Laboratory of Applied Health Sciences, Kyoto Prefectural University of Medicine, Kyoto, Japan; ${ }^{3}$ Department of Health and Sports, Faculty of Health and Medical Sciences, Kyoto Gakuen University, Kameoka, Japan; ${ }^{4}$ Central Research Institute for Physical Activity, Fukuoka University, Fukuoka, Japan; ${ }^{5}$ Department of Food and Health Sciences, Prefectural University of Kumamoto, Kumamoto, Japan; ${ }^{6}$ Laboratory of Healthy Longevity Research, National Institute of Health and Nutrition, National Institutes of Biomedical Innovation, Health and Nutrition, Tokyo, Japan
Correspondence: Yuya Watanabe Faculty of Health and Sports Science, Doshisha University, I-3 Tatara Miyakodani, Kyotanabe-shi, Kyoto 610-0394, Japan

Tel/fax+8I 774656720

Email yuwatana@mail.doshisha.ac.jp
Background: Skeletal muscle experiences both quantitative and qualitative changes with aging. Echo intensity (EI) of the skeletal muscle obtained by ultrasonography (US) has been considered to reflect muscle quality. This technique is noninvasive, easily accessible, and is relatively inexpensive than that of other imaging techniques such as computed tomography (CT). Previous studies have reported that EI is related to several physical performances. However, few studies have investigated the validity of EI against other imaging methods in case of imaging of the skeletal muscle. We compared quantitative and qualitative indices evaluated by the US and CT imaging systems to strengthen their validity.

Participants and methods: A total of 40 adults participated in this study: 19 young (10 men and 9 women; their mean $( \pm \mathrm{SD})$ age was $22.7 \pm 1.5$ years) and 21 older adults (13 men and 8 women; their mean age was $70.6 \pm 4.8$ years). Both thighs of each participant were evaluated using US and CT imaging systems.

Results: With respect to the US indices, the young group had significantly higher muscle thickness $(\mathrm{MT})$ of the front thigh $(P<0.001)$ and lower EI $(P=0.001)$ than that of the older group. With respect to the $\mathrm{CT}$ indices, the cross-sectional area (CSA) and mean CT value (Hounsfield unit [HU]) of the muscle were found to be significantly higher in the young group $(P<0.001)$ than that of the older group, whereas the percentage of low-density muscle area (\%LDMA) was found to be significantly higher in the older group $(P<0.001)$ than that of the young group. A significant, strong, and positive correlation was observed between MT and CSA, which reflects the muscle quantity $(r=0.774 ; P<0.001)$. With respect to the indices of muscle quality, we found significant and moderate correlations between EI and CT values $(r=-0.502 ; P<0.001)$ and between EI and \%LDMA $(r=0.441 ; P<0.001)$. However, these correlations were found to be decreased in the older group [between EI and CT value $(r=-0.363 ; P=0.018)$ and between EI and \%LDMA $(r=0.257 ; P=0.100)]$.

Conclusion: Results of this study indicate that the EI is moderately associated with muscle attenuation as assessed by CT, which means that higher EI at least partly reflects intramuscular lipid infiltration.

Keywords: echogenicity, ultrasound imaging, CT value, low-density muscle area, muscle composition, muscle quality

\section{Introduction}

Sarcopenia is a phenomenon in which there is gradual loss in the mass and function of the skeletal muscle with aging. ${ }^{1,2}$ It is considered as the primary or one of the 
major component(s) of frailty, especially physical frailty. Recent meta-analysis studies have reported that sarcopenia is associated with harmful health outcomes such as functional decline and mortality. ${ }^{3,4}$ It also triggers loss of independence and leads to increased medical-related costs. Therefore, prevention and/or improvement of sarcopenia is of utmost importance for the advanced human societies.

In addition to quantitative depletion of skeletal muscle, qualitative changes occur with aging. Typical age-related qualitative changes in skeletal muscle are decreasing number of Types I and II muscle fibers and decreasing size of Type II fibers, ${ }^{5,6}$ increasing inter or intramuscular adipose and connective tissues, ${ }^{7,8}$ and increased extracellular water molecules relative to muscle volume. ${ }^{9}$ These changes in the muscle composition are associated with decreased motor function and/or insulin resistance. ${ }^{10-12}$ Therefore, assessment of qualitative factors in the skeletal muscle is important when dealing with sarcopenia. However, in general, assessment of skeletal muscle mass using normal imaging methods, such as magnetic resonance imaging (MRI), computed tomography (CT), as well as dual energy X-ray absorptiometry (DXA) cannot reflect the qualitative status of skeletal muscle. Therefore, an evaluation method that can perform qualitative assessment of the skeletal muscle is highly warranted.

To evaluate the composition of the skeletal muscle, Hounsfield units (HU) in CT image (CT value) ${ }^{13}$ or MRI signal intensity can be used. ${ }^{14}$ However, it is difficult to obtain these values for many community-dwelling older people because of the need of special equipments and measurement burden (time, cost, and radiation exposure). Recently, echo intensity (EI), calculated from the images of skeletal muscular ultrasonography (US), has also been used to evaluate muscle composition. This technique is noninvasive, easily accessible, and relatively inexpensive than that of other imaging systems such as CT. Several groups of researchers have used EI as an index of skeletal muscle quality or composition. ${ }^{15-23}$ EI has been shown to be related to several physical performances. ${ }^{18-21}$ Furthermore, recent symposium reports introduced the effectiveness of skeletal muscular EI as an index of muscle quality. ${ }^{24,25}$

To establish the validity of EI, previous studies have compared EI obtained from US images with muscle fat content evaluated by MRI which revealed a moderate to strong correlation between them. ${ }^{26,27}$ However, to the best of our knowledge, no study has examined the association between EI and muscle attenuation as assessed by CT. Therefore, we compared quantitative and qualitative indices evaluated by US and CT imaging systems to strengthen the validity of EI as an indicator of muscle composition. We hypothesized that EI assessed by US is associated with muscle attenuation as assessed by CT.

\section{Participants and methods Study design}

In this study, we evaluated the thighs of both young and older participants using US and CT imaging systems and analyzed the cross-sectional images obtained as described elsewhere..$^{20,28,29}$ The sample size was determined based on two previous studies. ${ }^{26,27}$ The study protocol was approved by the Ethics Committee of Kyoto Prefectural University of Medicine (RBMR-E-382) and the Ethics Committee of Fukuoka University (11-04-01).

In this study, the indices of muscle quantity and quality obtained by each method (US and CT) were compared between young group and older group. In addition, the relationship between quantitative and qualitative indicators from both methods was examined. Each measurement was conducted on separate days. In this study, the mean interval between the two measurements was found to be 63.5 days (range: 6-117 days).

\section{Participants}

A total of 40 adults participated in this study: 19 young ( 10 men and 9 women; with mean $( \pm S D)$ age of $22.7 \pm 1.5$ years; range: $21-27$ years) and 21 older adults ( 13 men and 8 women; with mean age of $70.6 \pm 4.8$ years; range: $66-85$ years). All the participants were community-dwelling and lived independently without the need for any long-term care assistance. Young participants were requited at Fukuoka University, whereas older participants were requited at the Health Center in Nakagawa Town, Fukuoka prefecture, Japan. All participants were able to walk without an assistive device and had no history of lower limb trauma or surgery, no neuromuscular disorder, no acute or chronic diseases that impaired their strength and power, and had no severe dementia (which might influence the obtainment of informed consent). In addition, there were no participants with uncontrollable hypertension, diabetes, coronary heart disease, or hypothyroidism and were not on steroid medication. All participants were fully informed about the experimental procedures and the purpose of this study. All participants provided their written informed consent prior to their participation in this study.

\section{Ultrasound measurement}

The tissue thickness of the anterior compartment of both thighs was measured by B-mode US imaging, as described 
elsewhere. ${ }^{20}$ Transverse images were obtained with a B-mode US imaging device (SonoSite 180 Plus; SonoSite Japan, Tokyo, Japan) and a multi-frequency linear transducer (5-10 MHz). The gain was kept constant during all measurements and was not changed between participants. The measurements were performed with the participants in standing and completely relaxed position. The position of measurement was the midpoint between the anterior superior iliac spine and the lateral epicondyle of the femur. The transducer was positioned perpendicular to the longitudinal axis of the quadriceps femoris. The scanning head was pretreated with water-soluble transmission gel that provided acoustic contact without compressing the surface of the skin. A single investigator, who is a trained expert in the technical aspects of using the US machine, performed the measurements.

An electric caliper on frozen transverse US images was used to measure muscle and subcutaneous fat thickness (MT and FT, respectively). MT of the anterior compartment of the thigh was defined as the sum of thicknesses of the rectus femoris muscle and the vastus intermedius muscle. FT of the anterior compartment of the thigh was defined as the distance between the fascia of the rectus femoris muscle and the dermis. The measurements were repeated twice (a third measurement was performed in cases where there was a difference greater than $2 \mathrm{~mm}$ between the two measurements), and the median of these values was used in the analysis. The intraclass correlation coefficient (ICC) and mean coefficient of variation $(\mathrm{CV})$ for the repeated measurements were found to be respectively 0.991 and $0.965 \%$ for MT and 0.987 and $3.574 \%$ for FT.

From single stored images of US, the EI value was determined by gray-scale analysis using the standard histogram function in Adobe Photoshop Elements (Adobe Systems, San Jose, CA, USA). ${ }^{20} \mathrm{EI}$, as a muscle qualitative index, was defined as the mean pixel intensity in a region of interest (ROI). Each ROI included the rectus femoris muscle as much possible without any bone-surrounding fascia. The EI value in the ROI was expressed as a value between 0 (black) and 255 (white). The ICC and CV for the repeated measurements of EI were found to be 0.869 and $5.374 \%$, respectively.

\section{Computed tomography scan}

A CT scanner (Toshiba Multi-CT Aquilion TSX-101A Scanner, Toshiba Medical Systems, Tokyo, Japan) was used to evaluate the quantity and quality of the mid-thigh muscles of the participants. ${ }^{28,29}$ Scans of both thighs were performed at a peak voltage of $135 \mathrm{kVp}$ and at $400 \mathrm{~mA}$, with a scan time of 0.5 seconds and a $512 \times 512$ matrix. The data from six images, each $2 \mathrm{~mm}$ thick, from the midpoint between the anterior iliac crest and the superior border of the patella of the thigh were superimposed to create volume data of $10 \mathrm{~mm}$. All participants fasted for at least 3 hours but were allowed to consume water before the CT scan was performed. All CT scans were performed by the staff of Fukuseikai Hospital.

A cross-sectional area (CSA) and mean skeletal muscle Hounsfield unit (HU) value (CT value) of the mid-thigh were calculated using CT image analysis software on a Macintosh computer (OsiriX ver 3.3; OsiriX Foundation, Geneva, Switzerland). In addition, low-density skeletal muscle area (LDMA), a marker of lipid-rich skeletal muscle, was quantified within a $0-30 \mathrm{HU}$ attenuation window. ${ }^{30}$ We used CSA of the mid-thigh as an index of muscle size, whereas CT value and percentage of LDMA in CSA (\%LDMA) were used as indices of muscle quality.

\section{Statistical analyses}

Statistical analyses were performed using SPSS software (version 22.0; SPSS Japan, Tokyo, Japan). All values are reported as mean and SD. The differences in parameters between the young and older groups were detected using an unpaired $t$-test. The difference in sex ratio was analyzed using a chi-squared test. Correlations between variables were calculated using Pearson product-moment correlation coefficients. $P$-values $<0.05$ were defined a statistically significant.

\section{Results}

Table 1 shows the physical characteristics and muscle quantity and quality indices as determined by US and CT imaging systems in both groups. No significant differences were observed in the percentage of women between the two groups $(P=0.192)$. Figure 1 shows representative examples of US and CT images in young and older participants.

With respect to the anthropometric variables, participants in the young group were found to be taller than those of the older group $(P=0.001)$, although there was no significant difference in body weight. Furthermore, body mass index (BMI) was found to be significantly higher in the older group than that of the young group $(P=0.029)$. With respect to the US indices, participants in the young group were found to have significantly higher MT $(P<0.001)$ and lower EI $(P=0.001)$ than those of the older group. However, there was no significant difference in the FT between the two groups $(P=0.457)$. With respect to the $\mathrm{CT}$ indices, CSA and mean CT value were found to be significantly higher in the young group $(P<0.001)$, whereas \%LDMA was found to be 
Table I Physical characteristics and muscle quantity and quality indices of the participants

\begin{tabular}{|c|c|c|c|}
\hline Variables & $\begin{array}{l}\text { Young group } \\
(n=19,38 \text { legs) } \\
\text { mean } \pm \text { SD }\end{array}$ & $\begin{array}{l}\text { Older group } \\
(n=21,42 \text { legs) } \\
\text { mean } \pm S D\end{array}$ & $P$-value \\
\hline Age (years) & $22.7 \pm 1.5$ & $70.6 \pm 4.8$ & $<0.001$ \\
\hline $\begin{array}{l}\text { Number of } \\
\text { women }(\mathrm{n}(\%))\end{array}$ & $10(52.6 \%)$ & $8(38.1 \%)$ & 0.192 \\
\hline Height $(\mathrm{cm})$ & $160.0 \pm 5.9$ & $158.3 \pm 7.8$ & 0.001 \\
\hline Weight (kg) & $59.2 \pm 7.7$ & $57.8 \pm 8.6$ & 0.599 \\
\hline BMI $\left(\mathrm{kg} / \mathrm{m}^{2}\right)$ & $21.4 \pm 2.0$ & $23.0 \pm 2.4$ & 0.029 \\
\hline $\mathrm{MT}(\mathrm{mm})$ & $54.1 \pm 4.3$ & $45.2 \pm 5.6$ & $<0.001$ \\
\hline $\mathrm{FT}(\mathrm{mm})$ & $11.5 \pm 4.8$ & $10.6 \pm 5.2$ & 0.457 \\
\hline El & $15.6 \pm 3.3$ & $19.9 \pm 6.4$ & 0.001 \\
\hline Muscle CSA $\left(\mathrm{cm}^{2}\right)$ & $130.0 \pm 21.2$ & $100.9 \pm 19.4$ & $<0.001$ \\
\hline $\mathrm{CT}$ value $(\mathrm{HU})$ & $56.8 \pm 4.2$ & $51.1 \pm 4.3$ & $<0.001$ \\
\hline \%LDMA (\%) & $8.4 \pm 3.6$ & $|4.8 \pm 5|$. & $<0.001$ \\
\hline
\end{tabular}

Abbreviations: BMI, body mass index; CSA, cross-sectional area; $\mathrm{CT}$, computed tomography; El, echo intensity; FT, fat thickness of front thigh; HU, Hounsfield units; \%LDMA, percentage of low-density muscle area; MT, muscle thickness of front thigh.

significantly higher in the older group than that of the young group $(P<0.001)$.

Figure 2 shows the correlations between MT and CSA, between EI and CT value, and between EI and \%LDMA.
A significant, strong, and positive correlation was observed between MT and CSA reflecting muscle quantity ( $r=0.774$; $P<0.001)$. With respect to the muscle quality indices, there were significant and moderate correlations between EI and CT values $(r=-0.524 ; P<0.001)$ and between EI and \%LDMA values $(r=0.460 ; P<0.001)$. In addition, we examined the aforementioned correlations separately by age group (young or older group). The results demonstrated similar correlations in the young group, but the correlation coefficients were found to be decreased in the older group (Table 2). Although a significant but weak correlation was found between EI and CT values ( $r=-0.363 ; P=0.018)$, there was no significant correlation between EI and \%LDMA $(r=0.257 ; P=0.100)$ in the older group.

\section{Discussion}

In this study, following four important findings have been demonstrated: 1) significant age differences were detected in quantitative and qualitative indicators as determined by both methods; 2) significant correlation was obtained between MT of the front thigh and thigh muscle CSA; 3) significant correlations were obtained between EI and CT indices
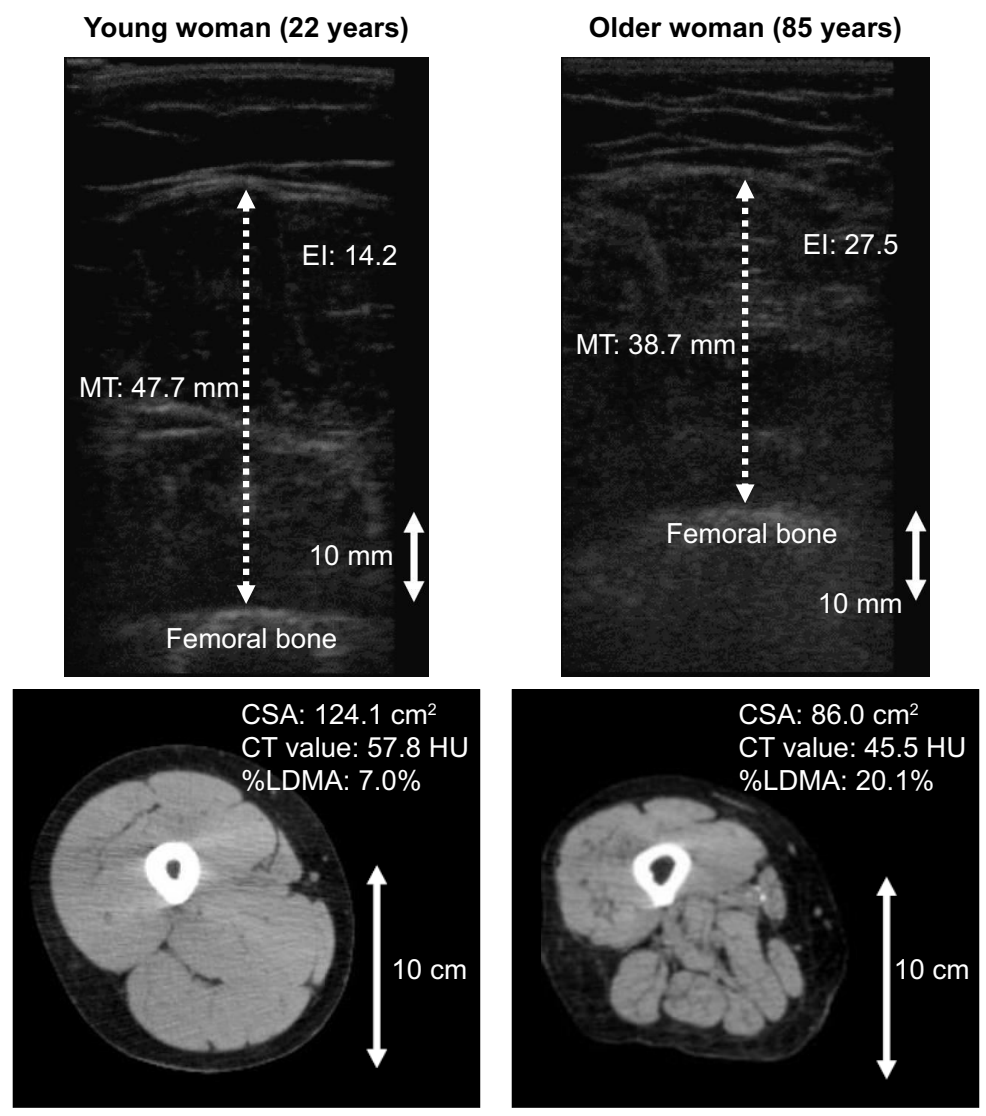

Figure I Representative examples of US (upper panels) and CT (lower panels) images of young (left panels) and older (right panels) participant.

Abbreviations: CSA, cross-sectional area of muscle; CT, computed tomography; El, echo intensity; HU, Hounsfield units; MT, muscle thickness of front thigh; US, ultrasonography; \%LDMA, percentage of low-density muscle area. 

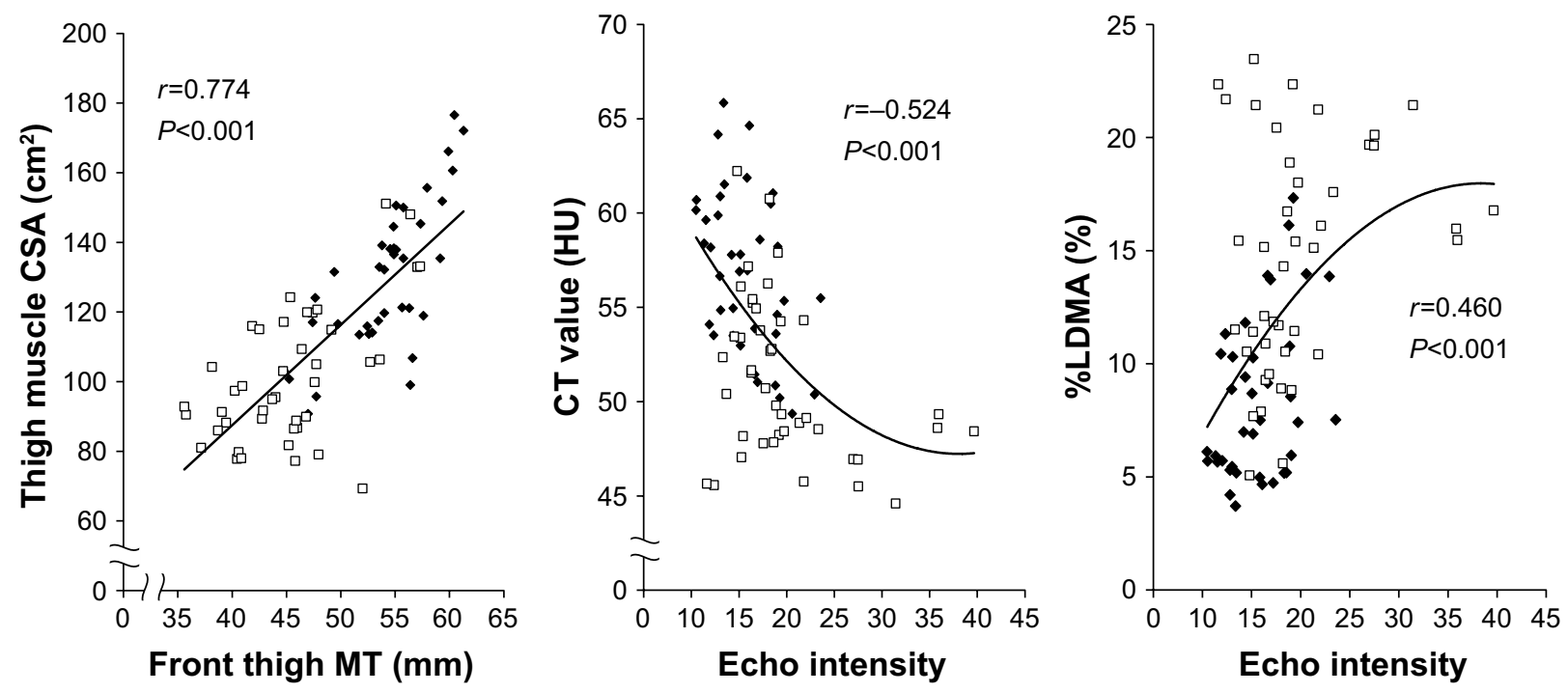

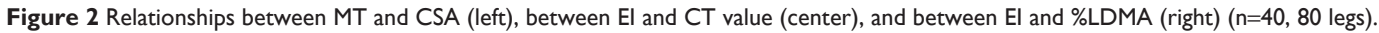
Note: Square $(\square)$ indicates older individuals and diamond $(\bullet)$ indicates young individuals.

Abbreviations: CSA, cross-sectional area; CT, computed tomography; El, echo intensity; HU, Hounsfield units; MT, muscle thickness; \%LDMA, percentage of low-density muscle area.

(CT value and \%LDMA); and 4) although their correlations were moderate, the correlations were found to be decreased in the older group. These results at least partly support our hypothesis with respect to the effectiveness of EI as an evaluation index of muscle quality related to muscle attenuation as assessed by CT.

Recently, EI, as determined by US imaging, has been recognized as a noninvasive, easily accessible, and a relatively inexpensive technique than that of other imaging systems such as $\mathrm{CT}$, in the evaluation of muscle composition. US images of the skeletal muscle of older individuals have a higher EI (looks whiter) than those of younger individuals.

Table 2 Correlation coefficients between US and CT methods in all, young, and older participants

\begin{tabular}{lll}
\hline Relationship & $\boldsymbol{r}$ & $\boldsymbol{P}$-value \\
\hline Between MT and CSA & & \\
All ( $\mathrm{n}=40,80$ legs) & 0.774 & $<0.00$ I \\
Young ( $\mathrm{n}=19,38$ legs) & 0.736 & $<0.00$ I \\
Older ( $\mathrm{n}=21$, 42 legs) & 0.574 & $<0.00$ I \\
Between El and CT value & & \\
All ( $\mathrm{n}=40,80$ legs) & -0.524 & $<0.00$ I \\
Young ( $\mathrm{n}=19,38$ legs) & -0.458 & 0.004 \\
Older ( $\mathrm{n}=21,42$ legs) & -0.363 & 0.018 \\
Between El and \%LDMA & & \\
All ( $\mathrm{n}=40,80$ legs) & 0.460 & $<0.00$ I \\
Young ( $\mathrm{n}=19,38$ legs) & 0.397 & 0.014 \\
Older ( $\mathrm{n}=21,42$ legs) & 0.257 & 0.100 \\
\hline
\end{tabular}

Notes: The relationship between EI and CT value and between El and \%LDMA in all participants are shown by a quadratic regression curve. Others are a linear regression.

Abbreviations: CSA, cross-sectional area of muscle; CT, computed tomography; El, echo intensity; MT, muscle thickness of front thigh; US, ultrasonography; \%LDMA, percentage of low-density muscle area.
It is generally thought that a high-homogeneity tissue such as the skeletal muscle is visualized as a low-intensity area (darker) on US, and a low-homogeneity tissue such as subcutaneous adipose is visualized as a high-intensity area (whiter). Thus, it is expected that enhanced EI in older people reflects age-related changes in their muscle composition. Several reports have shown qualitative changes in the skeletal muscle in older individuals such as increased fat and/or fibrous tissue $^{7,8}$ and relatively increased extracellular water molecules than that of young individuals. ${ }^{9}$ Some of these changes are thought to be related to the EI of the skeletal muscle. Following are the factors that may increase the EI of the skeletal muscle by inducing reflection of the US wave: 1) increase of noncontractile elements (fat and connective tissue) within muscle, ${ }^{32,33}$ 2) structural degeneration of extracellular matrix such as an increase in the concentration of collagen, ${ }^{34}$ and 3 ) decreased homogeneity of skeletal muscle fiber direction. ${ }^{35}$

Goodpaster et al reported a significant correlation between the skeletal muscle $\mathrm{CT}$ value of the mid-thigh and fat content of the vastus lateralis muscle evaluated by muscle biopsy in young-to-middle age individuals $(r=-0.58, P=0.02) .{ }^{30}$ Therefore, the mean HU value or \%LDMA of the skeletal muscle evaluated by $\mathrm{CT}$ have been used as indices of intramuscular fat mass. Although it is well known that $\mathrm{CT}$ value decreases with aging, the factors that affect $\mathrm{CT}$ value should be noted. The CT value of the skeletal muscle is influenced not only by an increase or decrease in the intramuscular fat content $(-30 \text { to }-190 \mathrm{HU})^{31}$ but also by the presence of water molecules within the muscle ( $0 \mathrm{HU})$. The tissue of skeletal muscle holds a large volume of water molecules partitioned 
into extracellular and intracellular compartments. Yamada et al reported that extracellular water molecules relative to the muscle volume increased with age. ${ }^{9}$ In this study, the correlation coefficients between $\mathrm{EI}$ and $\mathrm{CT}$ indices were found to differ between groups (Table 2). These results suggest that the factors influencing the EI and CT parameters are different. It is highly possible that the relative expansion of extracellular water content in the skeletal muscle affected the CT value in older participants. Furthermore, increased extracellular water molecules may be one of the primary reasons for the decrease in the correlation coefficients between EI and CT indices in the older group, although we cannot make a definitive conclusion owing to the lack of water distribution data. Changes in water distribution in muscle may lead to deformation of the extracellular matrix and cause reflection of the US wave. However, EI is not considered to be influenced by water within muscle. Although US imaging technique has some problems, such as measurement errors and compatibility of evaluation values, EI may be superior to CT in the evaluation of the proportion of noncontractile elements in muscle.

Previously, only two studies have investigated the association between EI, as assessed by US, and fat content within muscle, as evaluated by MRI. ${ }^{26,27}$ Young et al evaluated four lower-limb muscles (rectus femoris, biceps femoris, tibialis anterior, and medial gastrocnemius muscles) with US and MRI in 31 participants (29-61 years) and observed moderate to strong correlations between EI and intramuscular fat contents $(r=0.45-0.79) .{ }^{26}$ They also used equations to estimate the percentage of intramuscular fat from EI and FT as evaluated by US. ${ }^{26}$ More recently, Akima et al assessed relationships among EI, intramuscular fat content, as determined by MRI, and intramyocellular and extramyocellular lipids, as determined by ${ }^{1} \mathrm{H}$ magnetic resonance spectroscopy ( ${ }^{1} \mathrm{H}$ MRS) of vastus lateralis (VL) and biceps femoris (BF) muscles in 15 young (mean=20.9 years) and 15 older adults (mean=70.7 years). ${ }^{27}$ They found significant but moderate correlations between EI and intramuscular fat (VL: $r=0.404$; BF: $r=0.493$ ) and between EI and extramyocellular lipids (VL: $r=0.485$; BF: $r=0.648) .{ }^{27}$ However, there was no significant correlation between EI and intramyocellular lipids (VL: $r=0.060$; BF: $r=0.341$ ). ${ }^{27}$ Therefore, they concluded that enhanced EI in the US images of the skeletal muscle reflects extramyocellular adipose tissue and not intramyocellular adipose tissue. ${ }^{27}$ These two studies ${ }^{26,27}$ revealed the availability of EI as an index of intramuscular fat content by comparison with the MRI technique. Previously, Reimers et al investigated muscle echogenicity and biopsy samples of several muscles in human and concluded that enhanced
EI was primarily caused by an increased intramuscular lipid content. ${ }^{15}$ Our findings align with these previous reports. ${ }^{26,27}$ However, it should be noted that EI of the skeletal muscle may be affected by the deposition of fibrous connective tissue within the muscle. Experimental studies in animals have suggested a strong correlation between EI and interstitial fibrous tissue, which was measured in biopsy samples ( $r=0.87, P<0.001) .^{32}$ Therefore, this suggests that EI can be used as a comprehensive index of muscle composition and not just to evaluate intramuscular fat infiltration.

There were some limitations in this study. First, the interval between the two measurement types tended to be long (mean: 63.5 days). The reason is that all CT scans in this study were performed during the normal work of Fukuseikai Hospital. Our participants did not take part in any intervention program and were not instructed to change their physical activity and nutritional pattern in the duration between the two measurements. Therefore, we believe that the participants followed a similar lifestyle until the end of the two measurements. We also believe that muscle composition of the participants did not change between the two measurements. However, we cannot exclude the possibility of slight change, which may weaken the association between US and CT indices. Second, the ROIs of the two measurements were different in this study. The US measurement was conducted on the anterior compartment of the thigh, whereas the CT scan was performed on the whole thigh. This difference may influence the results of this study. However, the results of this study also imply that the EI of the rectus femoris muscle predicts the muscle composition of the whole thigh. Third, the midpoint of the thigh in the two measurements was different (US: between the anterior superior iliac spine and the lateral epicondyle of the femur; CT: between the anterior iliac crest and the superior border of the patella). The exact location is very important when comparing evaluation values by different methods. ${ }^{36}$ However, the target location of US measurement did not exactly match that of CT evaluation. The difference should be noted. Finally, EI value was evaluated from a single US image. This method has been used in our large-scale physical function measurement for communitydwelling older adults $(n=1,463) \cdot{ }^{37}$ A short time evaluation should be given a priority in fieldwork for older people. We evaluated EI from a single US image to investigate the validity of the technique used in the field work, although it may be less precise than the method of other research groups. In this study, significant correlations between the results of US and CT imaging were observed even with such limitations. We think that the results are important. 


\section{Conclusion}

In this study, we compared the quantitative and qualitative indices of the thigh skeletal muscle by using US and CT imaging systems in young and older participants. The results showed significant correlations between EI and CT values $(r=-0.524 ; P<0.001)$ and between EI and \%LDMA values $(r=0.460 ; P<0.001)$. However, the correlations were found to be decreased in the older participants potentially owing to changes in water distribution within the muscle.

In addition, our results indicate that EI of the skeletal muscle can be at least partly substituted for CT in the evaluation of muscle composition. Based on the merits of US imaging such as noninvasiveness, easy accessibility, and cost-effectiveness, we recommend that this technique can be useful in the evaluation of quality and quantity of the skeletal muscle for a wide range of people. In some cases, such as for older individuals and those with edema, EI obtained from US images may be an effective indicator of muscle quality than that of CT value or \%LDMA, although further investigation is needed.

\section{Acknowledgments}

The authors would like to thank all the participants of this study as well as Fukuseikai Hospital, Fukuoka, Japan for their technical assistance with CT examinations. We are also grateful to Dr Hiroaki Tanaka, Fukuoka University. This work was supported by JSPS KAKENHI to YW (grant nos 25750360 , 15K16531, 18K10873) and MI (grant no 26882056), and a grant from the Meiji Yasuda Life Foundation of Health and Welfare, Japan.

\section{Disclosure}

The authors report no conflicts of interest in this work.

\section{References}

1. Cruz-Jentoft AJ, Baeyens JP, Bauer JM, et al. Sarcopenia: European consensus on definition and diagnosis: Report of the European Working Group on Sarcopenia in Older People. Age Ageing. 2010;39(4):412-423.

2. Chen LK, Liu LK, Woo J, et al. Sarcopenia in Asia: consensus report of the Asian Working Group for Sarcopenia. J Am Med Dir Assoc. 2014;15(2):95-101.

3. Beaudart C, Zaaria M, Pasleau F, Reginster JY, Bruyère O. Health Outcomes of Sarcopenia: A Systematic Review and Meta-Analysis. PLoS One. 2017;12(1):e0169548.

4. Liu P, Hao Q, Hai S, Wang H, Cao L, Dong B. Sarcopenia as a predictor of all-cause mortality among community-dwelling older people: A systematic review and meta-analysis. Maturitas. 2017;103:16-22. Epub 2017 Apr 11.

5. Lexell J, Taylor CC, Sjöström M. What is the cause of the ageing atrophy? Total number, size and proportion of different fiber types studied in whole vastus lateralis muscle from 15 - to 83 -year-old men. $J$ Neurol Sci. 1988;84(2-3):275-294.
6. Lexell J. Human aging, muscle mass, and fiber type composition. J Gerontol A Biol Sci Med Sci. 1995;50:11-16.

7. Rice CL, Cunningham DA, Paterson DH, Lefcoe MS. Arm and leg composition determined by computed tomography in young and elderly men. Clin Physiol. 1989;9(3):207-220.

8. Overend TJ, Cunningham DA, Paterson DH, Lefcoe MS. Thigh composition in young and elderly men determined by computed tomography. Clin Physiol. 1992;12(6):629-640.

9. Yamada Y, Schoeller DA, Nakamura E, Morimoto T, Kimura M, Oda S. Extracellular water may mask actual muscle atrophy during aging. J Gerontol A Biol Sci Med Sci. 2010;65(5):510-516.

10. Goodpaster BH, Krishnaswami S, Resnick H, et al. Association between regional adipose tissue distribution and both type 2 diabetes and impaired glucose tolerance in elderly men and women. Diabetes Care. 2003;26(2):372-379.

11. Goodpaster BH, Thaete FL, Kelley DE. Thigh adipose tissue distribution is associated with insulin resistance in obesity and in type 2 diabetes mellitus. Am J Clin Nutr. 2000;71(4):885-892.

12. Addison O, Marcus RL, Lastayo PC, Ryan AS. Intermuscular fat: a review of the consequences and causes. Int J Endocrinol. 2014;2014: 309570-11. Epub 2014 Jan 8.

13. Goodpaster BH, Carlson CL, Visser M, et al. Attenuation of skeletal muscle and strength in the elderly: The Health ABC Study. $J$ Appl Physiol. 2001;90(6):2157-2165.

14. Kent-Braun JA, Ng AV, Young K. Skeletal muscle contractile and noncontractile components in young and older women and men. $J$ Appl Physiol. 2000;88(2):662-668.

15. Reimers K, Reimers CD, Wagner S, Paetzke I, Pongratz DE. Skeletal muscle sonography: a correlative study of echogenicity and morphology. J Ultrasound Med. 1993;12(2):73-77.

16. Pillen S, van Keimpema M, Nievelstein RA, Verrips A, van KruijsbergenRaijmann W, Zwarts MJ. Skeletal muscle ultrasonography: Visual versus quantitative evaluation. Ultrasound Med Biol. 2006;32(9): $1315-1321$.

17. Arts IM, Pillen S, Schelhaas HJ, Overeem S, Zwarts MJ. Normal values for quantitative muscle ultrasonography in adults. Muscle Nerve. 2010; 41(1):32-41.

18. Fukumoto Y, Ikezoe T, Yamada Y, et al. Skeletal muscle quality assessed from echo intensity is associated with muscle strength of middle-aged and elderly persons. Eur J Appl Physiol. 2012;112(4):1519-1525.

19. Cadore EL, Izquierdo M, Conceição M, et al. Echo intensity is associated with skeletal muscle power and cardiovascular performance in elderly men. Exp Gerontol. 2012;47(6):473-478.

20. Watanabe Y, Yamada Y, Fukumoto Y, et al. Echo intensity obtained from ultrasonography images reflecting muscle strength in elderly men. Clin Interv Aging. 2013;8:993-998. Epub 2013 Jul 25.

21. Rech A, Radaelli R, Goltz FR, da Rosa LH, Schneider CD, Pinto RS. Echo intensity is negatively associated with functional capacity in older women. Age. 2014;36(5):9708.

22. Yoshiko A, Kaji T, Sugiyama H, Koike T, Oshida Y, Akima H. Effect of 12-month resistance and endurance training on quality, quantity, and function of skeletal muscle in older adults requiring long-term care. Exp Gerontol. 2017;98:230-237. Epub 2017 Sep 11.

23. Yamada M, Kimura Y, Ishiyama D, et al. Differential Characteristics of Skeletal Muscle in Community-Dwelling Older Adults. $J$ Am Med Dir Assoc. 2017;18(9):807.e9-80807. Epub 2017 Jul 1.

24. Heymsfield SB, Gonzalez MC, Lu J, Jia G, Zheng J. Skeletal muscle mass and quality: evolution of modern measurement concepts in the context of sarcopenia. Proc Nutr Soc. 2015;74(4):355-366. Epub 2015 Apr 8.

25. Correa-de-Araujo R, Harris-Love MO, Miljkovic I, Fragala MS, Anthony BW, Manini TM. The Need for Standardized Assessment of Muscle Quality in Skeletal Muscle Function Deficit and Other AgingRelated Muscle Dysfunctions: A Symposium Report. Front Physiol. 2017;8:87.

26. Young HJ, Jenkins NT, Zhao Q, Mccully KK. Measurement of intramuscular fat by muscle echo intensity. Muscle Nerve. 2015;52(6):963-971. Epub 2015 Sep 7. 
27. Akima H, Hioki M, Yoshiko A, et al. Intramuscular adipose tissue determined by T1-weighted MRI at 3T primarily reflects extramyocellular lipids. Magn Reson Imaging. 2016;34(4):397-403. Epub 2015 Dec 30.

28. Yoshimura E, Kumahara H, Tobina T, et al. A 12-week aerobic exercise program without energy restriction improves intrahepatic fat, liver function and atherosclerosis-related factors. Obes Res Clin Pract. 2011; 5(3):e249-e257.

29. Yoshimura E, Kumahara H, Tobina T, et al. Lifestyle intervention involving calorie restriction with or without aerobic exercise training improves liver fat in adults with visceral adiposity. J Obes. 2014;2014: 197216-8. Epub 2014 Apr 17.

30. Goodpaster BH, Kelley DE, Thaete FL, He J, Ross R. Skeletal muscle attenuation determined by computed tomography is associated with skeletal muscle lipid content. J Appl Physiol. 2000;89(1):104-110.

31. Goodpaster BH, Thaete FL, Kelley DE. Thigh adipose tissue distribution is associated with insulin resistance in obesity and in type 2 diabetes mellitus. Am J Clin Nutr. 2000;71(4):885-992.

32. Pillen S, Tak RO, Zwarts MJ, et al. Skeletal muscle ultrasound: correlation between fibrous tissue and echo intensity. Ultrasound Med Biol. 2009;35(3):443-446.
33. Reimers CD, Schlotter B, Eicke BM, Witt TN. Calf enlargement in neuromuscular diseases: a quantitative ultrasound study in 350 patients and review of the literature. J Neurol Sci. 1996;143(1-2):46-56.

34. Kragstrup TW, Kjaer M, Mackey AL. Structural, biochemical, cellular, and functional changes in skeletal muscle extracellular matrix with aging. Scand J Med Sci Sports. 2011;21(6): 749-757.

35. Galbán CJ, Maderwald S, Stock F, Ladd ME. Age-related changes in skeletal muscle as detected by diffusion tensor magnetic resonance imaging. J Gerontol A Biol Sci Med Sci. 2007;62(4):453-458.

36. Willan PL, Ransome JA, Mahon M. Variability in human quadriceps muscles: quantitative study and review of clinical literature. Clin Anat. 2002; 15(2):116-128.

37. Watanabe Y, Yamada Y, Yokoyama K, et al. Comprehensive geriatric intervention program with and without weekly class-style exercise: research protocol of a cluster randomized controlled trial in KyotoKameoka Study. Clin Interv Aging. 2018;13:1019-1033.
Clinical Interventions in Aging

\section{Publish your work in this journal}

Clinical Interventions in Aging is an international, peer-reviewed journal focusing on evidence-based reports on the value or lack thereof of treatments intended to prevent or delay the onset of maladaptive correlates of aging in human beings. This journal is indexed on PubMed Central, MedLine,

\section{Dovepress}

CAS, Scopus and the Elsevier Bibliographic databases. The manuscript management system is completely online and includes a very quick and fair peer-review system, which is all easy to use. Visit http://www.dovepress. com/testimonials.php to read real quotes from published authors. 